\title{
Limiting Similarity Revisited
}

Péter Szabó (pszabo@angel.elte.hu)

Géza Meszéna (geza@angel.elte.hu)

\section{Approved by}

Ulf Dieckmann

Program Leader, ADN

September 2005

Interim Reports on work of the International Institute for Applied Systems Analysis receive only limited review. Views or opinions expressed herein do not necessarily represent those of the Institute, its National Member Organizations, or other organizations supporting the work. 


\section{IIASA STUDIES IN ADAPTIVE DYNAMICS No. 102}

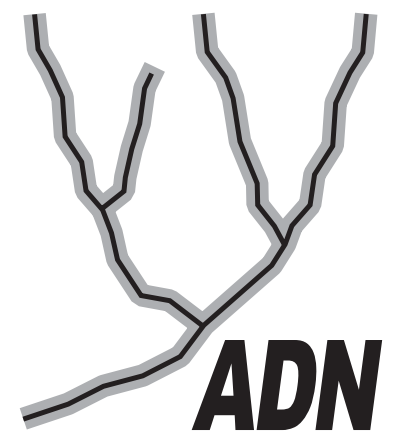

The Adaptive Dynamics Network at IIASA fosters the development of new mathematical and conceptual techniques for understanding the evolution of complex adaptive systems.

Focusing on these long-term implications of adaptive processes in systems of limited growth, the Adaptive Dynamics Network brings together scientists and institutions from around the world with IIASA acting as the central node.

Scientific progress within the network is collected in the IIASA Studies in Adaptive Dynamics series.

No. 1 Metz JAJ, Geritz SAH, Meszéna G, Jacobs FJA, van Heerwaarden JS: Adaptive Dynamics: A Geometrical Study of the Consequences of Nearly Faithful Reproduction. IIASA Working Paper WP-95-099 (1995). van Strien SJ, Verduyn Lunel SM (eds): Stochastic and Spatial Structures of Dynamical Systems, Proceedings of the Royal Dutch Academy of Science (KNAW Verhandelingen), North Holland, Amsterdam, pp. 183-231 (1996).

No. 2 Dieckmann U, Law R: The Dynamical Theory of Coevolution: A Derivation from Stochastic Ecological Processes. IIASA Working Paper WP-96-001 (1996). Journal of Mathematical Biology 34:579-612 (1996).

No. 3 Dieckmann U, Marrow P, Law R: Evolutionary Cycling of Predator-PreyInteractions: Population Dynamics and the Red Queen. IIASA Preprint (1995). Journal of Theoretical Biology 176:91-102 (1995).

No. 4 Marrow P, Dieckmann U, Law R: Evolutionary Dynamics of Predator-Prey Systems: An Ecological Perspective. IIASA Working Paper WP-96-002 (1996). Journal of Mathematical Biology 34:556-578 (1996).

No. 5 Law R, Marrow P, Dieckmann U: On Evolution under Asymmetric Competition. IIASA Working Paper WP-96-003 (1996). Evolutionary Ecology 11:485-501 (1997).

No. 6 Metz JAJ, Mylius SD, Diekmann O: When Does Evolution Optimize? On the Relation Between Types of Density Dependence and Evolutionarily Stable Life History Parameters. IIASA Working Paper WP-96-004 (1996).

No. 7 Ferrière R, Gatto M: Lyapunov Exponents and the Mathematics of Invasion in Oscillatory or Chaotic Populations. Theoretical Population Biology 48:126-171 (1995).

No. 8 Ferrière R, Fox GA: Chaos and Evolution. IIASA Preprint (1996). Trends in Ecology and Evolution 10:480485 (1995).

No. 9 Ferrière R, Michod RE: The Evolution of Cooperation in Spatially Heterogeneous Populations. IIASA Working Paper WP-96-029 (1996). The American Naturalist 147:692717 (1996).

No. 10 van Dooren TJM, Metz JAJ: Delayed Maturation in Temporally Structured Populations with Non-Equilibrium Dynamics. IIASA Working Paper WP-96-070 (1996). Journal of Evolutionary Biology 11:41-62 (1998).
No. 11 Geritz SAH, Metz JAJ, Kisdi É, Meszéna G: The Dynamics of Adaptation and Evolutionary Branching. IIASA Working Paper WP-96-077 (1996). Physical Review Letters 78:2024-2027 (1997).

No. 12 Geritz SAH, Kisdi É, Meszéna G, Metz JAJ: Evolutionary Singular Strategies and the Adaptive Growth and Branching of the Evolutionary Tree. IIASA Working Paper WP-96-114 (1996). Evolutionary Ecology 12:35-57 (1998).

No. 13 Heino M, Metz JAJ, Kaitala V: Evolution of Mixed Maturation Strategies in Semelparous Life-Histories: The Crucial Role of Dimensionality of Feedback Environment. IIASA Working Paper WP-96-126 (1996). Philosophical Transactions of the Royal Society of London Series B 352:1647-1655 (1997).

No. 14 Dieckmann U: Can Adaptive Dynamics Invade? IIASA Working Paper WP-96-152 (1996). Trends in Ecology and Evolution 12:128-131 (1997).

No. 15 Meszéna G, Czibula I, Geritz SAH: Adaptive Dynamics in a 2-Patch Environment: A Simple Model for Allopatric and Parapatric Speciation. IIASA Interim Report IR-97-001 (1997). Journal of Biological Systems 5:265-284 (1997).

No. 16 Heino M, Metz JAJ, Kaitala V: The Enigma of Frequency-Dependent Selection. IIASA Interim Report IR97-061 (1997). Trends in Ecology and Evolution 13:367-370 (1998).

No. 17 Heino M: Management of Evolving Fish Stocks. IIASA Interim Report IR-97-062 (1997). Canadian Journal of Fisheries and Aquatic Sciences 55:1971-1982 (1998).

No. 18 Heino M: Evolution of Mixed Reproductive Strategies in Simple Life-History Models. IIASA Interim Report IR-97063 (1997).

No. 19 Geritz SAH, van der Meijden E, Metz JAJ: Evolutionary Dynamics of Seed Size and Seedling Competitive Ability. IIASA Interim Report IR-97-071 (1997). Theoretical Population Biology 55:324-343 (1999).

No. 20 Galis F, Metz JAJ: Why Are There So Many Cichlid Species? On the Interplay of Speciation and Adaptive Radiation. IIASA Interim Report IR-97-072 (1997). Trends in Ecology and Evolution 13:1-2 (1998). 
No. 21 Boerlijst MC, Nowak MA, Sigmund K: Equal Pay for all Prisoners/ The Logic of Contrition. IIASA Interim Report IR-97-073 (1997). American Mathematical Society Monthly 104:303-307 (1997). Journal of Theoretical Biology 185:281-293 (1997).

No. 22 Law R, Dieckmann U: Symbiosis Without Mutualism and the Merger of Lineages in Evolution. IIASA Interim Report IR-97-074 (1997). Proceedings of the Royal Society of London Series B 265:1245-1253 (1998).

No. 23 Klinkhamer PGL, de Jong TJ, Metz JAJ: Sex and Size in Cosexual Plants. IIASA Interim Report IR-97-078 (1997). Trends in Ecology and Evolution 12:260-265 (1997).

No. 24 Fontana W, Schuster P: Shaping Space: The Possible and the Attainable in RNA Genotype-Phenotype Mapping. IIASA Interim Report IR-98-004 (1998). Journal of Theoretical Biology 194:491-515 (1998).

No. 25 Kisdi É, Geritz SAH: Adaptive Dynamics in Allele Space: Evolution of Genetic Polymorphism by Small Mutations in a Heterogeneous Environment. IIASA Interim Report IR-98-038 (1998). Evolution 53:993-1008 (1999).

No. 26 Fontana W, Schuster P: Continuity in Evolution: On the Nature of Transitions. IIASA Interim Report IR-98-039 (1998). Science 280:1451-1455 (1998).

No. 27 Nowak MA, Sigmund K: Evolution of Indirect Reciprocity by Image Scoring/ The Dynamics of Indirect Reciprocity. IIASA Interim Report IR-98-040 (1998). Nature 393:573-577 (1998). Journal of Theoretical Biology 194:561574 (1998).

No. 28 Kisdi É: Evolutionary Branching Under Asymmetric Competition. IIASA Interim Report IR-98-045 (1998). Journal of Theoretical Biology 197:149-162 (1999).

No. 29 Berger U: Best Response Adaptation for Role Games. IIASA Interim Report IR-98-086 (1998).

No. 30 van Dooren TJM: The Evolutionary Ecology of Dominance-Recessivity. IIASA Interim Report IR-98-096 (1998). Journal of Theoretical Biology 198:519-532 (1999).

No. 31 Dieckmann U, O'Hara B, Weisser W: The Evolutionary Ecology of Dispersal. IIASA Interim Report IR-98-108 (1998). Trends in Ecology and Evolution 14:88-90 (1999).

No. 32 Sigmund K: Complex Adaptive Systems and the Evolution of Reciprocation. IIASA Interim Report IR-98-100 (1998). Ecosystems 1:444-448 (1998).

No. 33 Posch M, Pichler A, Sigmund K: The Efficiency of Adapting Aspiration Levels. IIASA Interim Report IR-98103 (1998). Proceedings of the Royal Society London Series B 266:1427-1435 (1999).

No. 34 Mathias A, Kisdi É: Evolutionary Branching and Coexistence of Germination Strategies. IIASA Interim Report IR-99-014 (1999).

No. 35 Dieckmann U, Doebeli M: On the Origin of Species by Sympatric Speciation. IIASA Interim Report IR-99-013 (1999). Nature 400:354-357 (1999).

No. 36 Metz JAJ, Gyllenberg M: How Should We Define Fitness in Structured Metapopulation Models? Including an Application to the Calculation of Evolutionarily Stable Dispersal Strategies. IIASA Interim Report IR-99-019 (1999). Proceedings of the Royal Society of London Series B 268:499508 (2001)
No. 37 Gyllenberg M, Metz JAJ: On Fitness in Structured Metapopulations. IIASA Interim Report IR-99-037 (1999). Journal of Mathematical Biology 43:545-560 (2001).

No. 38 Meszéna G, Metz JAJ: Species Diversity and Population Regulation: The Importance of Environmental Feedback Dimensionality. IIASA Interim Report IR-99-045 (1999).

No. 39 Kisdi É, Geritz SAH: Evolutionary Branching and Sympatric Speciation in Diploid Populations. IIASA Interim Report IR-99-048 (1999).

No. 40 Ylikarjula J, Heino M, Dieckmann U: Ecology and Adaptation of Stunted Growth in Fish. IIASA Interim Report IR-99-050 (1999). Evolutionary Ecology 13:433-453 (1999).

No. 41 Nowak MA, Sigmund K: Games on Grids. IIASA Interim Report IR-99-038 (1999). Dieckmann U, Law R, Metz JAJ (eds): The Geometry of Ecological Interactions: Simplifying Spatial Complexity, Cambridge University Press, Cambridge, UK, pp. 135-150 (2000).

No. 42 Ferrière R, Michod RE: Wave Patterns in Spatial Games and the Evolution of Cooperation. IIASA Interim Report IR-99-041 (1999). Dieckmann U, Law R, Metz JAJ (eds): The Geometry of Ecological Interactions: Simplifying Spatial Complexity, Cambridge University Press, Cambridge, UK, pp. 318-332 (2000).

No. 43 Kisdi É, Jacobs FJA, Geritz SAH: Red Queen Evolution by Cycles of Evolutionary Branching and Extinction. IIASA Interim Report IR-00-030 (2000). Selection 2:161$176(2001)$.

No. 44 Meszéna G, Kisdi É, Dieckmann U, Geritz SAH, Metz JAJ: Evolutionary Optimisation Models and Matrix Games in the Unified Perspective of Adaptive Dynamics. IIASA Interim Report IR-00-039 (2000). Selection 2:193-210 (2001).

No. 45 Parvinen K, Dieckmann U, Gyllenberg M, Metz JAJ: Evolution of Dispersal in Metapopulations with Local Density Dependence and Demographic Stochasticity. IIASA Interim Report IR-00-035 (2000). Journal of Evolutionary Biology 16:143-153 (2003).

No. 46 Doebeli M, Dieckmann U: Evolutionary Branching and Sympatric Speciation Caused by Different Types of Ecological Interactions. IIASA Interim Report IR-00-040 (2000). The American Naturalist 156:S77-S101 (2000).

No. 47 Heino M, Hanski I: Evolution of Migration Rate in a Spatially Realistic Metapopulation Model. IIASA Interim Report IR-00-044 (2000). The American Naturalist 157:495$511(2001)$.

No. 48 Gyllenberg M, Parvinen K, Dieckmann U: Evolutionary Suicide and Evolution of Dispersal in Structured Metapopulations. IIASA Interim Report IR-00-056 (2000). Journal of Mathematical Biology 45:79-105 (2002).

No. 49 van Dooren TJM: The Evolutionary Dynamics of Direct Phenotypic Overdominance: Emergence Possible, Loss Probable. IIASA Interim Report IR-00-048 (2000). Evolution 54:1899-1914 (2000).

No. 50 Nowak MA, Page KM, Sigmund K: Fairness Versus Reason in the Ultimatum Game. IIASA Interim Report IR00-57 (2000). Science 289:1773-1775 (2000).

No. 51 de Feo O, Ferrière R: Bifurcation Analysis of Population Invasion: On-Off Intermittency and Basin Riddling. IIASA Interim Report IR-00-074 (2000). International Journal of Bifurcation and Chaos 10:443-452 (2000). 
No. 52 Heino M, Laaka-Lindberg S: Clonal Dynamics and Evolution of Dormancy in the Leafy Hepatic Lophozia Silvicola. IIASA Interim Report IR-01-018 (2001). Oikos 94:525-532 (2001).

No. 53 Sigmund K, Hauert C, Nowak MA: Reward and Punishment in Minigames. IIASA Interim Report IR-01-031 (2001). Proceedings of the National Academy of Sciences of the USA 98:10757-10762 (2001).

No. 54 Hauert C, De Monte S, Sigmund K, Hofbauer J: Oscillations in Optional Public Good Games. IIASA Interim Report IR-01-036 (2001).

No. 55 Ferrière R, Le Galliard J: Invasion Fitness and Adaptive Dynamics in Spatial Population Models. IIASA Interim Report IR-01-043 (2001). Clobert J, Dhondt A, Danchin E, Nichols J (eds): Dispersal, Oxford University Press, pp. 57-79 (2001).

No. 56 de Mazancourt C, Loreau M, Dieckmann U: Can the Evolution of Plant Defense Lead to Plant-Herbivore Mutualism. IIASA Interim Report IR-01-053 (2001). The American Naturalist 158:109-123 (2001).

No. 57 Claessen D, Dieckmann U: Ontogenetic Niche Shifts and Evolutionary Branching in Size-Structured Populations. IIASA Interim Report IR-01-056 (2001). Evolutionary Ecology Research 4:189-217 (2002).

No. 58 Brandt H: Correlation Analysis of Fitness Landscapes. IIASA Interim Report IR-01-058 (2001).

No. 59 Dieckmann U: Adaptive Dynamics of Pathogen-Host Interacations. IIASA Interim Report IR-02-007 (2002). Dieckmann U, Metz JAJ, Sabelis MW, Sigmund K (eds): Adaptive Dynamics of Infectious Diseases: In Pursuit of Virulence Management, Cambridge University Press, Cambridge, UK, pp. 39-59 (2002).

No. 60 Nowak MA, Sigmund K: Super- and Coinfection: The Two Extremes. IIASA Interim Report IR-02-008 (2002). Dieckmann U, Metz JAJ, Sabelis MW, Sigmund K (eds): Adaptive Dynamics of Infectious Diseases: In Pursuit of Virulence Management, Cambridge University Press, Cambridge, UK, pp. 124-137 (2002).

No. 61 Sabelis MW, Metz JAJ: Taking Stock: Relating Theory to Experiment. IIASA Interim Report IR-02-009 (2002). Dieckmann U, Metz JAJ, Sabelis MW, Sigmund K (eds): Adaptive Dynamics of Infectious Diseases: In Pursuit of Virulence Management, Cambridge University Press, Cambridge, UK, pp. 379-398 (2002).

No. 62 Cheptou P, Dieckmann U: The Evolution of SelfFertilization in Density-Regulated Populations . IIASA Interim Report IR-02-024 (2002). Proceedings of the Royal Society of London Series B 269:1177-1186(2002).

No. 63 Bürger R: Additive Genetic Variation Under Intraspecific Competition and Stabilizing Selection: A Two-Locus Study. IIASA Interim Report IR-02-013 (2002). Theoretical Population Biology 61:197-213 (2002).

No. 64 Hauert C, De Monte S, Hofbauer J, Sigmund K: Volunteering as Red Queen Mechanism for Co-operation in Public Goods Games. IIASA Interim Report IR-02-041 (2002). Science 296:1129-1132 (2002).

No. 65 Dercole F, Ferrière R, Rinaldi S: Ecological Bistability and Evolutionary Reversals under Asymmetrical Competition. IIASA Interim Report IR-02-053 (2002). Evolution 56:1081-1090 (2002).
No. 66 Dercole F, Rinaldi S: Evolution of Cannibalistic Traits: Scenarios Derived from Adaptive Dynamics. IIASA Interim Report IR-02-054 (2002). Theoretical Population Biology 62:365-374 (2002).

No. 67 Bürger R, Gimelfarb A: Fluctuating Environments and the Role of Mutation in Maintaining Quantitative Genetic Variation. IIASA Interim Report IR-02-058 (2002). Genetical Research 80:31-46 (2002).

No. 68 Bürger R: On a Genetic Model of Intraspecific Competition and Stabilizing Selection. IIASA Interim Report IR02-062 (2002). Amer. Natur. 160:661-682 (2002).

No. 69 Doebeli M, Dieckmann U: Speciation Along Environmental Gradients. IIASA Interim Report IR-02-079 (2002). Nature 421:259-264 (2003).

No. 70 Dercole F, Irisson J, Rinaldi S: Bifurcation Analysis of a Prey-Predator Coevolution Model. IIASA Interim Report IR-02-078 (2002). SIAM Journal on Applied Mathematics 63:1378-1391 (2003).

No. 71 Le Galliard J, Ferrière R, Dieckmann U: The Adaptive Dynamics of Altruism in Spatially Heterogeneous Populations. IIASA Interim Report IR-03-006 (2003). Evolution 57:1-17 (2003).

No. 72 Taborsky B, Dieckmann U, Heino M: Unexpected Discontinuities in Life-History Evolution under SizeDependent Mortality. IIASA Interim Report IR-03-004 (2003). Proceedings of the Royal Society of London Series B 270:713-721 (2003).

No. 73 Gardmark A, Dieckmann U, Lundberg P: LifeHistory Evolution in Harvested Populations: The Role of Natural Predation. IIASA Interim Report IR-03-008 (2003). Evolutionary Ecology Research 5:239-257 (2003).

No. 74 Mizera F, Meszéna G: Spatial Niche Packing, Character Displacement and Adaptive Speciation Along an Environmental Gradient. IIASA Interim Report IR-03-062 (2003). Evolutionary Ecology Research 5:363-382 (2003).

No. 75 Dercole F: Remarks on Branching-Extinction Evolutionary Cycles. IIASA Interim Report IR-03-077 (2003). Journal of Mathematical Biology 47:569-580 (2003).

No. 76 Hofbauer J, Sigmund K: Evolutionary Game Dynamics. IIASA Interim Report IR-03-078 (2003). Bulletin of the American Mathematical Society 40:479-519 (2003).

No. 77 Ernande B, Dieckmann U, Heino M: Adaptive Changes in Harvested Populations: Plasticity and Evolution of Age and Size at Maturation. IIASA Interim Report IR03-058 (2003). Proceedings of the Royal Society of London Series B-Biological Sciences 271:415-423 (2004).

No. 78 Hanski I, Heino M: Metapopulation-Level Adaptation of Insect Host Plant Preference and Extinction-Colonization Dynamics in Heterogeneous Landscapes. IIASA Interim Report IR-03-028 (2003). Theoretical Population Biology 63:309-338 (2003).

No. 79 van Doorn G, Dieckmann U, Weissing FJ: Sympatric Speciation by Sexual Selection: A Critical Re-Evaluation. IIASA Interim Report IR-04-003 (2004). American Naturalist 163:709-725 (2004).

No. 80 Egas M, Dieckmann U, Sabelis MW: Evolution Restricts the Coexistence of Specialists and Generalists - the Role of Trade-off Structure. IIASA Interim Report IR-04-004 (2004). American Naturalist 163:518-531 (2004). 
No. 81 Ernande B, Dieckmann U: The Evolution of Phenotypic Plasticity in Spatially Structured Environments: Implications of Intraspecific Competition, Plasticity Costs, and Environmental Characteristics. IIASA Interim Report IR-04-006 (2004). Journal of Evolutionary Biology 17:613-628 (2004).

No. 82 Cressman R, Hofbauer J: Measure Dynamics on a One-Dimensional Continuous Trait Space: Theoretical Foundations for Adaptive Dynamics. IIASA Interim Report IR04-016 (2004).

No. 83 Cressman R: Dynamic Stability of the Replicator Equation with Continuous Strategy Space. IIASA Interim Report IR-04-017 (2004).

No. 84 Ravigné V, Olivieri I, Dieckmann U: Implications of Habitat Choice for Protected Polymorphisms. IIASA Interim Report IR-04-005 (2004). Evolutionary Ecology Research 6:125-145 (2004).

No. 85 Nowak MA, Sigmund K: Evolutionary Dynamics of Biological Games. IIASA Interim Report IR-04-013 (2004). Science 303:793-799 (2004).

No. 86 Vukics A, Asbóth J, Meszéna G: Speciation in Multidimensional Evolutionary Space. IIASA Interim Report IR-04-028 (2004). Physical Review 68:041-903 (2003).

No. 87 de Mazancourt C, Dieckmann U: Trade-off Geometries and Frequency-dependent Selection. IIASA Interim Report IR-04-039 (2004). American Naturalist 164:765-778 (2004).

No. 88 Cadet CR, Metz JAJ, Klinkhamer PGL: Size and the Not-So-Single Sex: disentangling the effects of size on sex allocation. IIASA Interim Report IR-04-084 (2004). American Naturalist 164:779-792 (2004).

No. 89 Rueffler C, van Dooren TJM, Metz JAJ: Adaptive Walks on Changing Landscapes: Levins' Approach Extended. IIASA Interim Report IR-04-083 (2004). Theoretical Population Biology 65:165-178 (2004).

No. 90 de Mazancourt C, Loreau M, Dieckmann U: Understanding Mutualism When There is Adaptation to the Partner. IIASA Interim Report IR-05-016 (2005). Journal of Ecology 93:305-314 (2005).

No. 91 Dieckmann U, Doebeli M: Pluralism in Evolutionary Theory. IIASA Interim Report IR-05-017 (2005). Journal of Evolutionary Biology 18:1209-1213 (2005).
No. 92 Doebeli M, Dieckmann U, Metz JAJ, Tautz D: What We Have Also Learned. IIASA Interim Report IR-05-018 (2005). Evolution 59:691-695 (2005).

No. 93 Egas M, Sabelis MW, Dieckmann U: Evolution of Specialization and Ecological Character Displacement of Herbivores Along a Gradient of Plant Quality. IIASA Interim Report IR-05-019 (2005). Evolution 59:507-520 (2005).

No. 94 Le Galliard J, Ferrière R, Dieckmann U: Adaptive Evolution of Social Traits: Origin, Trajectories, and Correlations of Altruism and Mobility. IIASA Interim Report IR05-020 (2005). American Naturalist 165:206-224 (2005).

No. 95 Doebeli M, Dieckmann U: Adaptive Dynamics as a Mathematical Tool for Studying the Ecology of Speciation Processes. IIASA Interim Report IR-05-022 (2005). Journal of Evolutionary Biology 18:1194-1200 (2005).

No. 96 Brandt H, Sigmund K: The Logic of Reprobation: Assessment and Action Rules for Indirect Reciprocity. IIASA Interim Report IR-04-085 (2004). Journal of Theoretical Biology 231:475-486 (2004).

No. 97 Hauert C, Haiden N, Sigmund K: The Dynamics of Public Goods. IIASA Interim Report IR-04-086 (2004). Discrete and Continuous Dynamical Systems - Series B 4:575587 (2004).

No. 98 Meszéna G, Gyllenberg M, Jacobs FJA, Metz JAJ: Link Between Population Dynamics and Dynamics of Darwinian Evolution. IIASA Interim Report IR-05-026 (2005). Physical Review Letters 95:Article 078105 (2005).

No. 99 Meszéna G: Adaptive Dynamics: The Continuity Argument. IIASA Interim Report IR-05-032 (2005). Journal of Evolutionary Biology 18:1182-1185 (2005).

No. 100 Brännström NA, Dieckmann U: Evolutionary Dynamics of Altruism and Cheating Among Social Amoebas. IIASA Interim Report IR-05-039 (2005). Proceedings of the Royal Society London Series B 272:1609-1616 (2005).

No. 101 Meszéna G, Gyllenberg M, Pasztor L, Metz JAJ: Competitive Exclusion and Limiting Similarity: A Unified Theory. IIASA Interim Report IR-05-040 (2005).

No. 102 Szabo P, Meszéna G: Limiting Similarity Revisited. IIASA Interim Report IR-05-050 (2005).

Issues of the IIASA Studies in Adaptive Dynamics series can be obtained at www.iiasa.ac.at/Research/ADN/Series.html or by writing to adn@iiasa.ac.at. 


\section{Contents}

Abstract.19. 2




\title{
Limiting similarity revisited
}

\author{
Péter Szabó ${ }^{1 *}$ and Géza Meszéna ${ }^{2}$ \\ 1,2 Department of Biological Physics, Eötvös University, Pázmány Péter stny. 1/A, H-1117, Budapest, \\ Hungary.
}

E-mail addresses:

${ }^{1}$ pszabo@angel.elte.hu

${ }^{2}$ geza@angel.elte.hu

* Corresponding author. Phone: +36 137227 86, Fax: +361372 2757 


\begin{abstract}
We reinvestigate the validity of the limiting similarity principle via numerical simulations of the Lotka-Volterra model. A Gaussian competition kernel is employed to describe decreasing competition with increasing difference in a one-dimensional phenotype variable. The simulations are initiated by a large number of species, evenly distributed along the phenotype axis. Exceptionally, the Gaussian carrying capacity supports coexistence of all species, initially present. In case of any other, distinctly different, carrying capacity functions, competition resulted in extinction of all, but a few species. A comprehensive study of classes of fractal-like carrying capacity functions with different fractal exponents was carried out. The average phenotype differences between surviving species were found to be roughly equal to the competition width. We conclude that, despite the existence of exceptional cases, the classical picture of limiting similarity and niche segregation is a good rule of thumb for practical purposes.
\end{abstract}

KEY WORDS: limiting similarity, niche segregation, interspecific competition, LotkaVolterra 


\section{INTRODUCTION}

Limiting similarity is a central, but controversial tenet of community ecology. The seminal paper of MacArthur and Levins (1967) was among the most influential theoretical papers in ecology. It stated that phenotype difference on the scale of the competition width is required between coexisting species. Since then, the assertion and the related figure became an indispensable part of any ecology textbook (Begon et al. 1996, Case 2000, Krebs 2001).

While the empirical relevance of limiting similarity was clearly demonstrated in several studies (see e.g. Schluter 2000a,b), its wider context, "competition theory", has gradually fallen out of favor since its inception in the sixties. It has become the prevailing view that importance of competition, and henceforth of limiting similarity, was originally overestimated; other types of interaction, like predation and disturbance, has a nonnegligible role in shaping communities (Begon et al. 1996). On the other hand, even more recently, other researchers stress that the effect of predation is very analogous to resource limitation; an attempt to revival of niche theory as a "central organizing aspect of ecology" was made on this basis (Leibold 1995).

Decline of competition theory overshadows the fact that the real prediction of it has never been made sufficiently clear. Competitive exclusion (Gause 1934, Hardin 1960) is often interpreted in the narrow, but mathematically clear, sense as a statement that the number of coexisting species cannot be larger than the number of resources, or "limiting factors" (e.g. Levin 1970, Armstrong and McGehee 1980). Partitioning of a resource continuum cannot be discussed this way, as the continuum represents an infinite 
number of different resources. Then, we either have a limiting similarity type statement, or competitive exclusion predicts nothing (cf. Rosenzweig 1995).

MacArthur \& Levins (1967) used the Lotka-Volterra competition model for demonstrating limiting similarity in resource partitioning. However, the more detailed analysis of the model by May and MacArthur (1972) established no clear lower bound of similarity. While May (1973) rescued the principle by referring to environmental fluctuation as a necessary ingredient of the limiting similarity argument, the very same result signaled the end of the road for limiting similarity according to Rosenzweig (1995): “... the result (limiting similarity) was quicksand that trapped the energies of community ecologists for more than ten years and nearly killed the subdiscipline".

Surprisingly, the most powerful mathematical counterexample for limiting similarity is not widely recognized in the ecological literature. Roughgarden (1979) demonstrated, that even a continuum of species is able to coexist in the Lotka-Volterra model. While this effort was intended to describe polymorphism within a species, it can be interpreted also in a community ecology context, as recognized by Maynard Smith and Szathmáry (1995).

Investigations going beyond the Lotka-Volterra model leaded to no firm conclusion, either (Abrams 1983, 1988). On the one hand, it seems to be clear that some kind of limit of similarity must exist in any model. On the other hand, no general result of this type has emerged.

More recently, following the lead by Sasaki and Elner (1995), Gyllenberg and Meszéna (2004) showed that any model, which allows continuous coexistence, is necessarily structurally unstable, i.e. an arbitrarily small modification of the model might 
be able to destroy the continuous coexistence. The continuous coexistence in Roughgarden's model will not survive a small perturbation of the carrying capacity curve. Meszéna et al. (submitted) showed that similarity of coexisting species decreases the tolerance of the assemblage towards the external environmental parameters. That is, similarity decreases the chance of coexistence. While these qualitative analytical results are very general, they do not predict any specific lower bound of similarity.

After many pros and cons, the single most important question, i.e. whether limiting similarity has any practical relevance, has remained unsolved. Here we intend to check the expectation that coexisting species should differ roughly according to their competition width. More specifically, assuming Gaussian competition functions with a standard deviation $\sigma$, can we generally expect, as a rule of thumb, $2 \sigma$ distances between coexisting species? We resort to a comparative study to answer this question. We investigate the cases, which support limiting similarity, and the cases, which defy it, in the same context. As both the existence and the non-existence of limiting similarity were originally demonstrated in the Lotka-Volterra model, we use this framework also. We repeat the same numerical experiment with different choices for the carrying capacity function. The Gaussian one, corresponding to Roughgarden's counter-example with the continuous coexistence, will be considered as the reference case.

\section{MODEL DEFINITION}

We start from the familiar Lotka-Volterra competition model for several species, which we write in the form 


$$
\frac{d n_{i}}{d t}=\alpha_{i} n_{i}\left(K_{i}-\sum_{j=1}^{L} a_{i j} n_{j}\right),
$$

where $L$ stands for the number of species, $i \in\{1,2, \ldots, L\}$ and $n_{i}$ denotes the density of the $i^{\text {th }}$ one. The elements of the competition matrix are denoted by $a_{i j} ; a_{i i}=1$ is assumed for all $i . K_{i}$ is the carrying capacity for species $i$. As the constant $\alpha_{i}$ is unimportant for our purpose, $\alpha_{i}=1$ will be chosen for each species.

Each species is characterized by a phenotype variable $x_{i}$. Then, $K_{i}$ and $a_{i j}$ are determined by the phenotype and the difference between phenotypes, respectively. Eq. (1) takes the form

$$
\frac{d n_{i}}{d t}=n_{i}\left(K\left(x_{i}\right)-\sum_{j=1}^{L} a\left(x_{i}, x_{j}\right) n_{j}\right) .
$$

We suppose decreasing competition with increasing phenotype difference, according to the usual Gaussian form

$$
a(x, y)=\exp \frac{-(x-y)^{2}}{2 \sigma^{2}}
$$

The twice standard deviation $2 \sigma$ of this Gaussian will be referred to as competition width. The carrying capacity function $K\left(x_{i}\right)$ will be specified in each example separately.

When the number of species is large and the difference between neighboring phenotypes is small, we use the continuous approximation

$$
\frac{d n(x)}{d t}=n(x)\left(K(x)-\int a(x, y) n(y) d y\right)
$$


where $n(x)$ stands for the density of species with phenotype $x$. Then the equilibrium condition reads as

$$
K\left(x_{i}\right)=\sum_{j=1}^{L} a\left(x_{i}, x_{j}\right) n_{j},
$$

or

$$
K(x)=\int a(x, y) n(y) d y .
$$

In equilibrium, these equations should hold for all species present in non-zero density.

For each choice for the carrying capacity function $K(x)$, we integrated Eq. (2) numerically with time steps of $\Delta t=0.1$. Simulations were initiated with 1001 species, evenly distributed within the phenotype interval $[-1,1]$ with equal densities of 0.01 . The outcome of competition was evaluated at $t=10000$. At this time point the growth rates were wery close to zero. The number of coexisting species was assessed by counting species that were present and exhibited nonnegative growth at $t=10000$. This criteria was applied to exclude populations which were bound to extinction, but eventually existed at that time. 
RESULTS

Roughgarden's example for continuous coexistence

Roughgarden's (1979) example employs the Gaussian carrying capacity function

$$
K(x)=\exp \frac{-x^{2}}{2 \omega^{2}}
$$

where $\omega$ denotes the half-width of this curve. Then the Gaussian species distribution

$$
n(x)=\frac{\omega / \sigma}{\sqrt{2 \pi\left(\omega^{2}-\sigma^{2}\right)}} \exp \frac{-x^{2}}{2\left(\omega^{2}-\sigma^{2}\right)}
$$

is an equilibrium solution of Eq. (4), provided that $\omega>\sigma$. In this case, infinitely many arbitrarily similar species may coexist along the phenotype axis.

We reproduced this analytical result numerically (Fig. 1). All of the populations, that were present initially, survived. In agreement with Eq. (8), the equilibrium distribution of the population densities followed a Gaussian curve. Increased competition width $\sigma$ led to a narrower distribution of densities. Obviously, when $\omega<\sigma$, i.e. when the carrying capacity is narrower than the competition width, only the species $x=0$ survives (not shown).

Other simple carrying capacity functions: no continuous coexistence

The rectangular function

$$
K(x)=\left\{\begin{array}{lll}
c, & \text { if } & x \in(-b ; b) \\
0, & \text { if } & x \notin(-b ; b)
\end{array}\right.
$$


which is zero outside an interval, is the next simple choice for the carrying capacity curve. In contrast to Roughgarden's case, most of the populations go extinct. Only a very limited number of species coexist at equilibrium (Fig. 2).

The average phenotype difference between adjacent survivors can be calculated by dividing the total phenotype interval with the number of coexisting species. As plotted on Fig. 3, this difference increased with $\sigma$ linearly with great accuracy. The steepness of the linear regression line was found to be 1.82 . That is, in agreement with the classical expectation, the coexisting species were spaced roughly by the competition width $2 \sigma$.

It is easy to interpret this result in qualitative terms. The two species, which are located at the two ends of the livable range, gain advantage from the lack of competitors on one of their sides. Then, competition by these high-density species causes extinction within their range of competition. Two empty ranges emerge which, in turn, give advantage to two species, located at the inner ends of the empty ranges. And so on, a discrete distribution emerges.

The perturbation, that renders the coexistence of infinitely many strategies impossible does not have to be so abrupt. We get similar results if $K(x)$ is a sum of two Gaussian functions as

$$
K(x)=\exp \frac{-x^{2}}{2 \omega_{1}^{2}}+\alpha \exp \frac{-(x-\mu)^{2}}{2 \omega_{2}^{2}}
$$

with $\omega_{1}>\sigma>\omega_{2}$. The first Gaussian, when alone, would maintain a continuous coexistence. In contrast, the second one supports a single phenotype, namely $x=\mu$. Fig. 4 demonstrates the emerging species distribution. The second term of Eq. (10) gives an advantage to species $x=\mu$. The high density of this species causes extinction of each 
species within its range of competition. Then, the empty ranges on both sides of species $x=\mu$ give advantage to the two species next to these ranges. And so on, the competitive advantages and disadvantages build up gradually and a discrete species distribution emerges.

The realistic case: Fractal-like carrying capacity functions

While the smooth Gaussian function in the role of the carrying capacity curve leads to continuous coexistence, a function with abrupt changes seems to result in limiting similarity in the usual sense. These extremes cannot tell us, however, what is the typical situation.

As a representative of a more natural function, we investigate fractal-like perturbation of the Gaussian carrying capacity. We chose

$$
K(x)=\exp \frac{-x^{2}}{2 \omega^{2}}(1+f(x))
$$

where the perturbation term

$$
f(x)=k \sum_{i=1}^{L} \beta_{i} \cos \left(\gamma i x+\varphi_{i}\right)
$$

consists of periodic components with random phases. The parameters $k$ and $\gamma$ are constants. The amplitude of the $i^{\text {th }}$ periodic component is

$$
\beta_{i}=1 / i^{v} .
$$


The phases $\varphi_{i}$ were chosen randomly for each simulation run. The exponent $\eta$ characterizes the fractal properties. $\eta=0$ for a white noise; Brownian motion is characterized by $\eta=1$.

We stress that, since $\varphi_{i}$ s remain constant during a single run of the simulation, the random choice of them does not introduce stochasticity into the dynamics. Instead, this randomization ensures that each simulation uses a different carrying capacity curve, characterized by a common fractal exponent. Accordingly, each data point in Figures 5 and 6 represents an average over a class of models.

Fig. 5 shows the results with $\eta=0,1,1.5$ respectively. All simulations ended up with survival of a limited number of species. The number of species at equilibrium and, consequently, average phenotype distances change with $\eta$. The number of coexisting species was averaged over 50 simulations, differing in the random phases, for each $\eta$ and $\sigma$ combination (Fig. 6).

Distances increase with $\sigma$ in all cases and the slope of the fitted line remains in the range $1.9 \sigma-2.6 \sigma$. The departure from the linear dependence on Fig. 6 is related to the fact that $\sigma$ is not small, compared to the total phenotype interval, at the right end of the horizontal scale. 


\section{DISCUSSION}

In this paper we have reconsidered the age-old problem of limiting similarity in a Lotka-Volterra model context. The need for this re-evaluation came from the fact that in different investigations the very same model was used for both the justification and falsification of this principle. Initiating the simulations with many equally abundant species, closely packed along the phenotype variable, we observed whether a limiting similarity type of species distribution shows up. In line with theoretical predictions, a yes-and-no picture emerged. As the issue seemed to be related to the smooth-or-not nature of the carrying capacity function, we investigated "natural", i.e. fractal-like, functions with different fractal exponents. We conclude that, in spite of the existence of exceptional cases, the classical picture of limiting similarity prevails, by and large. Especially, as a rule of thumb, the typical distance between coexisting species is near to the competition width $2 \sigma$. This result seems to be quite robust: We tested very different carrying capacity functions, including families of fractal-like ones.

Nevertheless, the details of the final distribution of the species in a specific simulation do not seem to be very regular. The surviving species are not exactly equidistant. Their abundances apparently depend on the exact shape of the carrying capacity curve as well as on which species survived. In most of the cases, the initial distribution is so far from the final one, that there is no reason to suppose that the limiting-similarity-type outcome depends on the initial distribution. On the other hand, in a specific simulation there is no reason to expect the exactly same final distribution for a different initial one. 
In a specific simulation run, any species either equilibrates with a finite abundance, or dies out. Its growth rate converges to zero in the first case, but remains negative in the second one. That is, in the final state the growth rates of all extinct phenotypes are negative. Consequently, the resulting species assemblage is stable against invasion when any phenotype, which is not present, is (re)introduced in small density (cf. Jansen and Mulder 1999, Bonsall et al. 2004). On the other hand, the evolutionarily stable community/assemblage needs not be unique, i.e. it may depend on the initial conditions.

Obviously, our initial condition is far from being realistic. In the real life, there is no such supply of an almost continuous set of species. Our point exactly is that limiting similarity emerges even from starting so irrealistically far from that outcome.

Our simulation results are consistent with the empirical findings. On the one hand, character displacement studies (Schluter 2000a,b) demonstrated segregation of coexisting species in resource related traits. Note, that similarity in the environmental requirements is also necessary for coexistence (cf. Leibold 1995, Chase and Leibold 2003), so investigations based of phylogenetic relationships (e.g. Webb 2000) will not necessarily demonstrate any correlation between coexistence and dissimilarity. On the other hand, the specific arrangement of species of a community is influenced by many factors, including the initial conditions, and is not predictable via simple rules.

Limiting similarity is not without exceptions. In some sense the non-existence of an absolute and model-independent lower bound of similarity is trivial; in a parameterrich model one can always adjust the parameters to equalize the growth rates of the species. Consequently, any set of strategies may be able to coexist, irrespective of their 
similarity. The real issue of coexistence starts when one refuses to fine-tune parameters. That is, the real question is whether the coexistence is robust against parameter changes. This triviality was noted first by May and MacArthur (1972), May (1973). They varied the carrying capacities of two, or three, species. The smaller the interspecific competition, the wider the range of the carrying capacities, which enabled coexistence, was. As interpecific competition is supposedly related to the ecological difference between species, this result means that the coexistence becomes more robust with increasing difference. That is, limiting similarity is a quantitative issue. Similarity does not exclude coexistence, but makes it less likely. This conclusion has been generalized beyond the Lotka-Voltarra context by Meszéna et al. (submitted).

Decreased competition between dissimilar species has a regulating effect on their coexistence. Suppose that a perturbation affects species $A$ adversely, but not $B$. Then the population size of $A$ starts to decline. If the intraspecific competition is larger than the interspecific one, the decreased population size of $A$ improves the living conditions of $A$ in a larger extent, than that of $B$. This, in turn, compensates species $A$ for the relative disadvantage it has gained by the original perturbation. Large niche overlap reduces this regulatory effect as both species gain roughly the same advantage from the decline of one of them.

The problem of continuous coexistence is a related issue. For any given form of the competition kernel $a(x, y)$ and for any prescribed species distribution $n(x)$, the equilibrium condition (Equ. (6)) determines a carrying capacity function $K(x)$, which allows the coexistence with this specific species distribution. However, as coexistence of similar strategies is possible only for a narrow range of parameters, one may reasonably 
presume that coexistence of a continuum of species should be sensitive to an arbitrarily small perturbation. In fact, this is the case.

Structural instability of Roughgarden's example for continuous coexistence was essentially noted already by Sasaki and Ellner (1995). Their context was entirely different; they considered mixed strategies in a fluctuating environment. Nevertheless, their criterion for an ESS mixing distribution was identical to the equilibrium condition for coexisting strategies in the Lotka-Volterra model. Consequently, their proof of the structural instability of any ESS, which mixes a continuum of pure strategies, translates to the structural instability of any Roughgarden's type of continuous coexistence. Geritz (1995, 1999) and Meszéna and Szathmáry (2001) provided additional models in which continuous coexistence appears, but only under structurally unstable circumstances. Gyllenberg and Meszéna (2004) generalized these results be proving the structural instability of any model, which allows continuous coexistence.

Haccou and Iwasa $(1995,1998)$ demonstrated a kind of smoothness in the abrupt loss of the continuous solution for the mixed strategy problem of Sasaki and Ellner (1995). For small perturbations, the emerging discrete distribution follows closely the original continuous one. No similar general analysis is available for the coexistence problem. Still, it is sensible to suspect that the transition from continuous to discrete coexistence is smooth, again. That is, small perturbation of Roughgarden's model probably allows coexistence of discrete, but very similar, strategies. One may conjecture that the minimal distance between coexisting species grows up from zero continuously with the increasing departure from the strictly Gaussian carrying capacity function. We did not attempt to follow this transition. Instead, we compared the strictly Gaussian case 
with the distinctly different ones and studied, what determines the lower bound of similarity. In all cases we tried, we found that the minimal phenotype difference is of the order of the competition width.

The mechanism leading to limiting similarity is transparent in the simple cases we presented. Local maxima of the carrying capacity curve gives advantage to some phenotypes. In turn, these favored phenotypes impose competitive burden on the neighboring types along the phenotype axis via competition. That is, the not-very-smooth nature of the carrying capacity curve is essential for limiting similarity. In this context, it was essential to study choices somewhere in between the very smooth and the sharply changing: the fractals.

We employed fractal functions to mitigate a serious problem of mathematical ecology. In the "strategic" level of modeling (cf. Czárán 1998) the model ingredients are chosen according to their simplicity, instead of their empirical fidelity. The expectation here is, that the simpler the choice is, the lesser the danger of introducing artefactual details. Unfortunately, the ingredient functions, which are the simplest choices from mathematical point of view, are often very artificial. For instance, they are either unnaturally smooth, like a Gaussian function, or unnaturally discontinuous, like a step function.

Fractals and fractal-like functions are ubiquitous in nature (Mandelbrot 1983). If a shape is influenced by many processes, probably the system will not be dominated by a single, or a few, characteristic scales (Beran 2004). A scale-independent, self-similar pattern may emerge as a result. Such shape is represented by an autocorrelation function, which decays according to a power law. The "fractal" exponent of the power law 
characterizes the relative strength of the shorter and longer correlations. Self-similar patterns have been reported in many biological systems at all level of the organization (Burrough 1981, Liebovitch and Sullivan 1987, Shlesinger and West 1991, Gunnarsson 1992, Harte et al. 1999). The carrying capacity function is a result of a complicated interplay between the ecological interactions and the physiological constraints, i.e. the genotype-phenotype map. The process is likely to involve many random components acting on multiple time scales, leading to multiscaled randomness (Hausdorff 1996). As there is no reason to expect any characteristic scale in such a carrying capacity function, it is reasonable to endow it with fractal-like properties, instead.

The fractal exponent $v$ characterizes the relative contributions of the different scales. The larger the exponent is, the smaller the amplitude of small scale perturbations. That is, a smaller $v$ represents a more rugged carrying capacity curve, while a larger one represents a smoother one. Varying the fractal exponent and choosing different phases in Eq. (12) allows us to sweep through a representative variety of possible carrying capacity functions. It is remarkable that our findings were essentially independent of the fractal exponent; fractal-like carrying capacity functions with different exponents have uniformly led to limiting similarity with roughly $(2.3 \pm 0.3) \sigma$ distance between coexisting species, i.e. slightly higher than competition width.

The Lotka-Volterra model is often criticized as "phenomenological", as opposed to the mechanistic models. Notwithstanding the success of mechanistic level of modeling (cf. Tilman 1982), it did not lead any consistent understanding on the issue of limited similarity. In particular, investigations hinted mechanism-dependence neither for the validity of the limiting similarity principle nor for the specific lower bound of similarity. 
In the contrary, the universality across different mechanisms (e.g. competition and predation) was stressed by Leibold (1995), Chase and Leibold (2003). Moreover, no principal difference between the phenomenological and mechanistic model is expected because the Lotka-Volterra model can be derived from a mechanistic underpinning (Yodzis 1989). Moreover, in any model one can locally linearize the density-dependence, which leads to a Lotka-Volterra model. Consequently, any issue, like linear stability and robustness, which can be studied via small perturbations, should be the same in the original model and in its Lotka-Volterra approximation.

While Abrams (1983), Yodzis (1989) stress the model dependence of limiting similarity, analytic investigations (Gyllenberg and Meszéna 2005, Meszéna et al. submitted) testify for the generality of some basic issues. First, simple counter-examples for limiting similarity exist already in the very model, which was used to demonstrate the principle. Second, both structural instability of continuous coexistence and decreasing robustness of coexistence for increasing similarity is proven generally. This is the context, in which detailed numerical investigations make sense: We have reasons to hope for the general relevance of our conclusions.

In particular, competition kernels, other than the Gaussian, are not expected to lead to essentially different results. It is clear, that the carrying capacity curve, which allows continuous coexistence, can be constructed for any competition function via the equilibrium equation (Eq. (6)). An infinitesimal perturbation of this carrying capacity will destroy the continuous coexistence, again (Gyllenberg and Meszéna 2004). Note that the standard deviation is not necessarily a good measure of the competition width for competition functions other than the Gaussian (Yodzis 1989). 
The misleading exceptionality of Roughgarden's model exhibits a serious methodological problem of ecological modeling. Nevertheless, the careful investigation of the Lotka-Volterra model in the context of analytical investigations provides a strong case for the practical relevance of the principle of limiting similarity.

\section{ACKNOWLEDGEMENTS}

We thanks Mats Gyllenberg, Patsy Haccou, Yoh Iwasa, Mathew Leibold, Hans Metz, Liz

Pásztor and Akira Sasaki for discussions. This work was financed from grants OTKA T033097, T049689 and NWO-OTKA 048.011.039. 


\section{BIBLIOGRAPHY}

Abrams, P. 1983. The theory of limiting similarity. - Annual Review of Ecology and Systematics 14: 359-376.

Abrams, P. 1988. How should resources be counted? - Theor. Popul. Biol. 33: 226-242.

Armstrong, R. A. and McGehee, R. 1980. Competitive exclusion. - American Naturalist 115: $151-170$.

Begon, M., Harper, J. L. and Townsend, C.R. 1996. Ecology. - Blackwell Science, Oxford, pp. 265, 300 .

Beran, J. 1984. Statistics for long-memory processes. - Chapman \& Hall, New York.

Bonsall, M. B., Jansen, V. A. A. and Hassel, M. P. 2004. Life history trade-offs assemble ecological guilds. - Science 306: 111-114.

Burrough, P. A. 1981. Fractal dimensions of landscapes and other environmental data. Nature 294: 240-242.

Case, T. J. 2000. An illustrated guide to theoretical ecology. - Oxford Univ. Press, Oxford, p. 374. 
Czárán, T. 1998. Spatiotemporal models of population and community dynamics. Chapman \& Hall, London.

Gause, G. F. 1934. The struggle for existence. - Williams and Wilkins, Baltimore.

Geritz, S. A. H.1995. Evolutionary stable seed polymorphism and small scale spatial variation in seedling density. - American Naturalist 146: 685-707.

Geritz, S. A. H., van der Meijden, E. and Metz, J. A. J. 1999. Evolutionary dynamics of seed size and seedling competitive ability. - Theor. Popul. Biol. 55: 324-343.

Gunnarsson, B. 1992. Fractal dimension of plants and body size distribution in spiders. Funct. Ecol. 6: 636-641.

Gyllenberg, M. and Meszéna, G. 2005. On the impossibility of coexistence of infinitely many strategies. - Journal of Mathematical Biology. 50: 133-160.

Haccou, P. and Iwasa, Y. 1995. Optimal mixed strategies in stochastic environments. Theor. Popul. Biol. 47: 212-243.

Haccou, P. and Iwasa Y. 1998. Robustness of optimal mixed strategies. - J. Math. Biol. 36: $485-496$. 
Hardin, G. 1960. The competitive exclusion principle. - Science 131: 1292-1297.

Harte, J., Kinzig, A. and Green, J. 1999. Self-similarity in the distribution and abundance of species. - Science 284: 334-336.

Hausdorff, J. M. and Peng, C. K. 1996. Multiscaled randomness: A possible source of 1/f noise in biology. - Physical Review E. 54: 2154-2155.

Jansen, V. A. A. and Mulder, S. E. E. 1999. Evolving biodiversity. - Ecology Letters 2: $379-386$

Krebs, C. J. 2001. Ecology, the Experimental Analysis of Distribution and Abundance, 5th ed. - Benjamin Cummins, San Francisco, p. 197.

Leibold, M. A. 1995. The niche concept revisited: mechanistic models and community context. - Ecology 76: 1371-1382.

Chase, J. M. and Leibold, M. A. 2003. Ecological niches: linking classical and contemporary approaches. - The University of Chicago Press, Chicago.

Levin, S. M. 1970. Community equilibria and stability, and an extension of the competitive exclusion principle. - American Naturalist 104: 413-423. 
Liebovitch, L. S. and Sullivan, J. M. 1987. Fractal analysis of a voltage-dependent potassium channel from cultured mouse hippocampal neurons. - Biophysical Journal 52: 979-988.

MacArthur, R. and Levins, R. 1967. The limiting similarity, convergence, and divergence of coexisting species. - American Naturalist 101: 377-385.

Mandelbrot, B. B. 1983. The fractal geometry of nature. - W. H. Freeman and Co., New York.

May, R. M. 1973. Stability and complexity in model ecosystems. - Princeton Univ. Press, Princeton.

May, R. M. and MacArthur, R. H. 1972. Niche overlap as a function of environmental variability. - Proc. Nat. Acad. Sci. 69: 1109-1113.

Maynard Smith, J. and Szathmáry, E. 1995. The Major Transitions in Evolution. - W. H. Freeman Press, Oxford, p. 163.

Meszéna, G. and Szahmáry, E. 2001. Adaptive dynamics of parabolic replicators. Selection 2: 147-159. 
Meszéna, G., Gyllenberg, M., Pásztor, L. and Metz, J. A. J. (submitted) Competitive exclusion and limiting similarity: a unified theory. - Theoretical Population Biology.

Rosenzweig, M. 1995. Species diversity in space and time. - Cambridge Univ. Press, Cambridge, pp. 6, 127.

Roughgarden, J. 1979. Theory of population genetics and evolutionary ecology: an introduction. - Macmillan Publishing Company, New York, pp. 534-536.

Sasaki, A. and Ellner, S. 1995. The evolutionarily stable phenotype distribution in a random environment. - Evolution 49: 337-350.

Schluter, D. 2000a. Ecological character displacement in adaptive radiation. - American Naturalist 156: S4-S16.

Schluter, D. 2000b. The ecology of adaptive radiation. - Oxford University Press, Oxford.

Shlesinger, M. F. and West, B. J. 1991. Complex fractal dimension of the bronchial tree. - Phys. Rev. Lett. 67: 2106-2108.

Tilman, D. 1982. Resource equilibrium and community structure. - Princeton University Press, Princeton. 
Webb, C. O. 2000. Exploring the phylogenetic structure of ecological communities: An example for rain forest trees. - American Naturalist 156: 145-155.

Yodzis, P. 1989. Introduction to theoretical ecology. - Harper and Row, New York, pp. $118-122$ 


\section{FIGURE LEGENDS:}

FIGURE 1. Species distribution with Gaussian carrying capacity function for different competition widths $2 \sigma$. On each plot, dashed line depicts the shape of the carrying capacity curve, normalized to a given height. Black region denotes the population distribution. Variance of the bell-shaped species distribution decreases with increasing competition width. Parameters: $\omega=0.3 ; \sigma=0.04,0.08,0.16$ in sub-figures a, b, c, respectively.

FIGURE 2. Species distribution with rectangular carrying capacity function for different competition widths $2 \sigma$. Only a finite number of species coexist. The number of coexisting species decreases with increasing $\sigma$. Parameters: $b=0.6, c=5, \sigma=0.04,0.08$, 0.16 in sub-figures $a, b, c$ respectively.

FIGURE 3. Phenotype difference between adjacent survivors as a function of $\sigma$ and the fitted linear regression line (solid line) for rectangular carrying capacity. Phenotype difference values are calculated as the number of species at equilibrium divided by the length of the phenotype interval. The steepness of the linear regression line is 1.82 .

FIGURE 4. Species distribution for different competition widths $2 \sigma$, when the carrying capacity function is composed from two Gaussians. Competition width increases from left to right; $\sigma=0.04,0.08,0.16$ in subfigures $a, b, c$ respectively. Other parameters: $\omega_{1}=0.3, \omega_{2}=0.03, \alpha=0.1, \mu=-0.3$. 
FIGURE 5. Species distribution with fractal-like carrying capacity function for different fractal dimensions $\eta$ and competition widths $2 \sigma$. The $\eta$ value increases from the top down: (a-c) $\eta=0$, (d-f) $\eta=1.0$, (g-i) $\eta=1.5$. $\sigma$ increases from left to right for each $\eta$ value; (a, d, g) $\sigma=0.02,(b, e, h) \sigma=0.04$, (c, f, i) $\sigma=0.08 . \omega=0.3$ in all cases.

FIGURE 6. Average phenotype differences between adjacent survivors $\mathrm{p}$ as a function of $\sigma$ with fractal-like $K(x)$. Difference values were averaged over 50 simulations with different random choices for the phases. Other parameters: $\omega=0.3$, (a) $\eta=0$, (b) $\eta=1.0$, (c) $\eta=1.5$. A linear regression line (solid line) was calculated for each $\eta$ (data points, denoted by empty circles, were ignored). Slopes of regression lines are 2.64, 1.97, 1.99, respectively. 
Szabó and Meszéna, page 28

Figure 1.
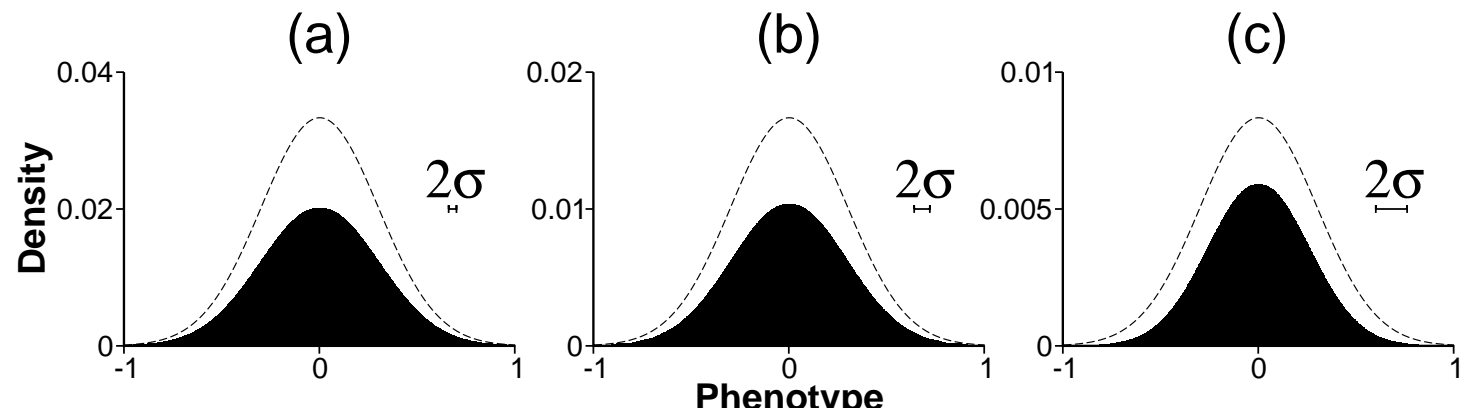
Szabó and Meszéna, page 29

Figure 2.

(a)

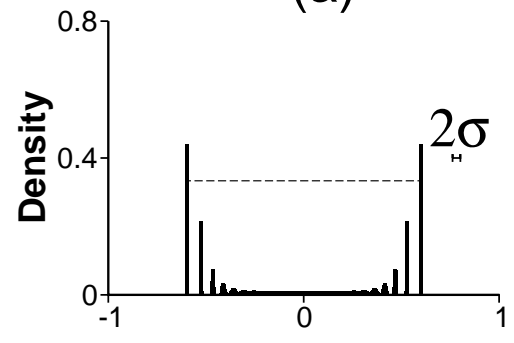

(b)

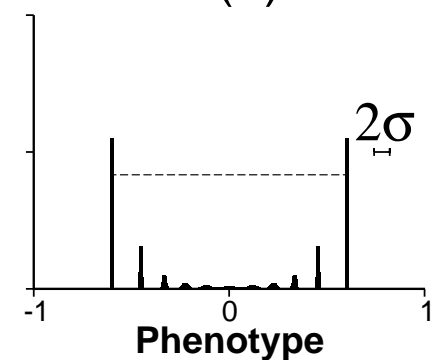

(c)

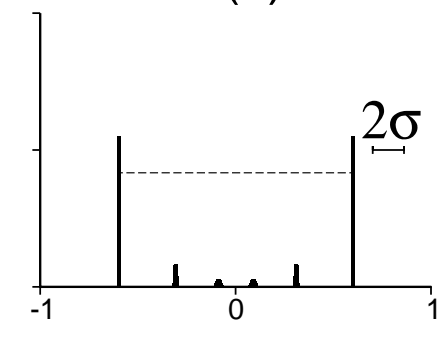


Szabó and Meszéna, page 30

Figure 3.

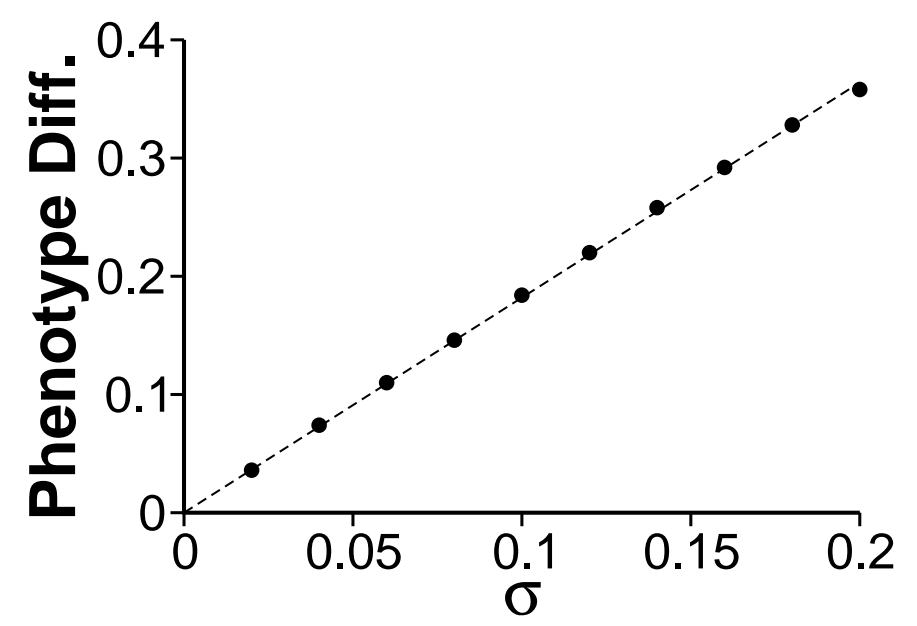


Szabó and Meszéna, page 31

Figure 4.

(a)

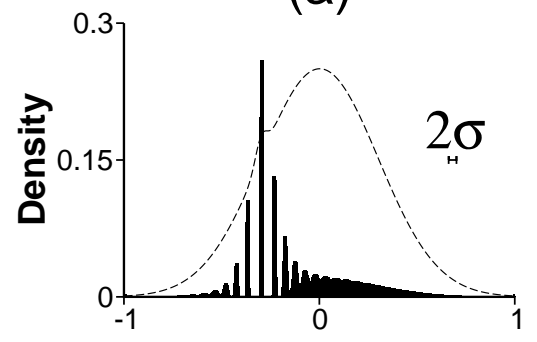

(b)

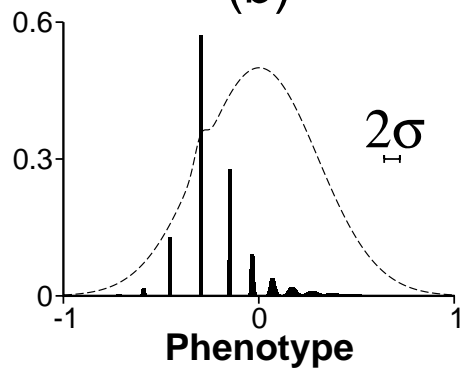

(c)

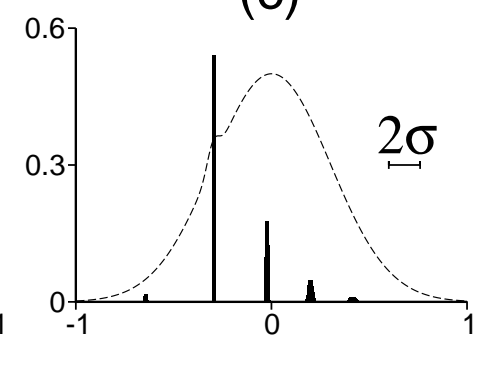


Figure 5.

(a)

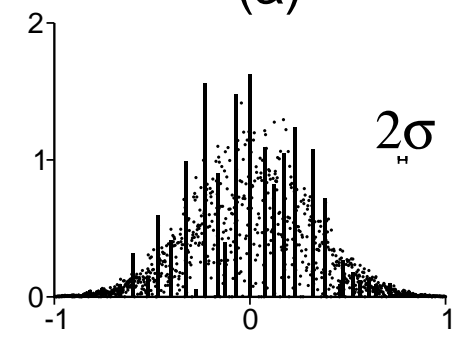

(d)

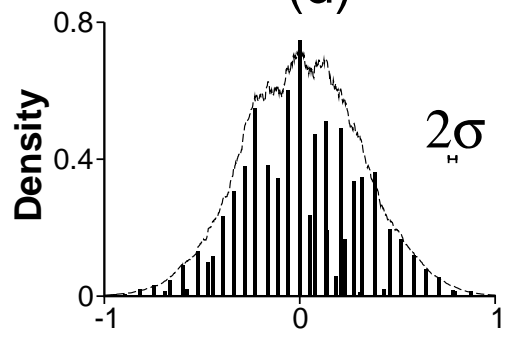

(g)

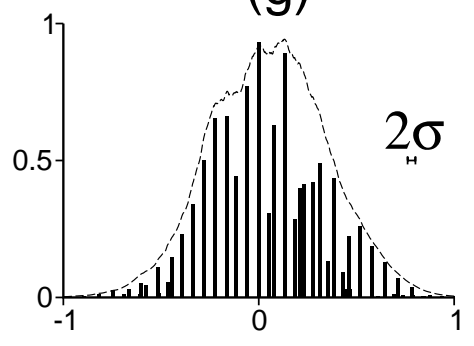

(b)

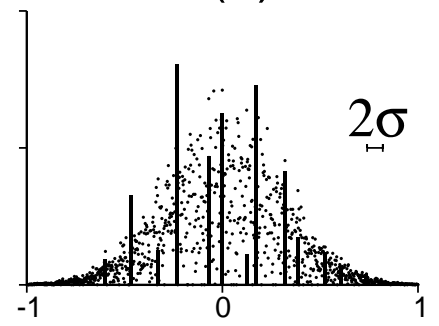

(e)

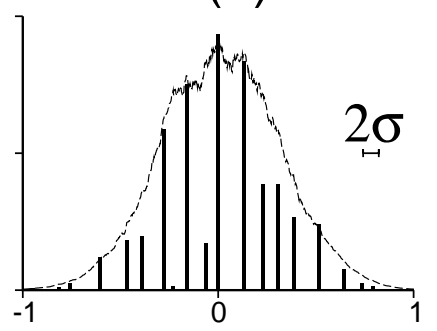

(h)

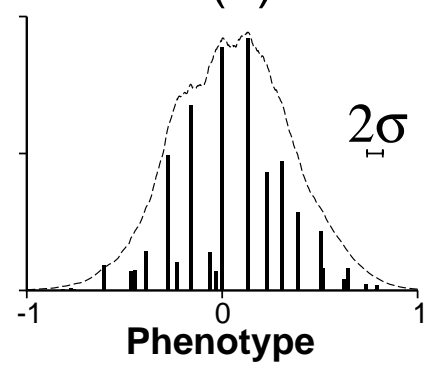

(c)

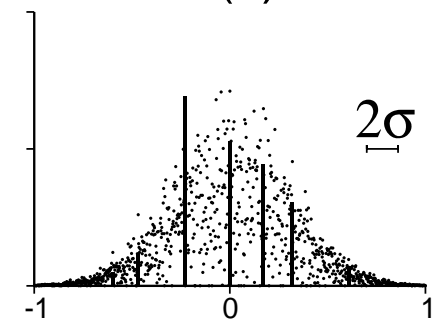

(f)

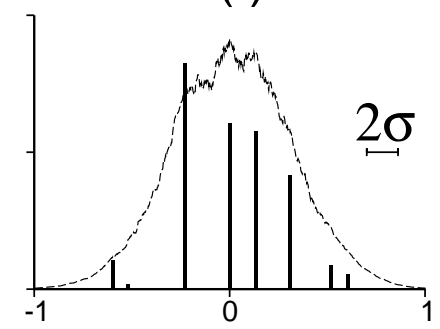

(i)

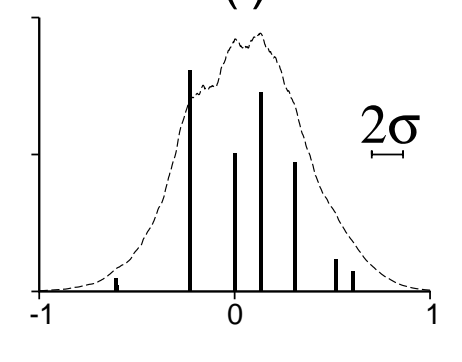


Szabó and Meszéna, page 33

Figure 6.
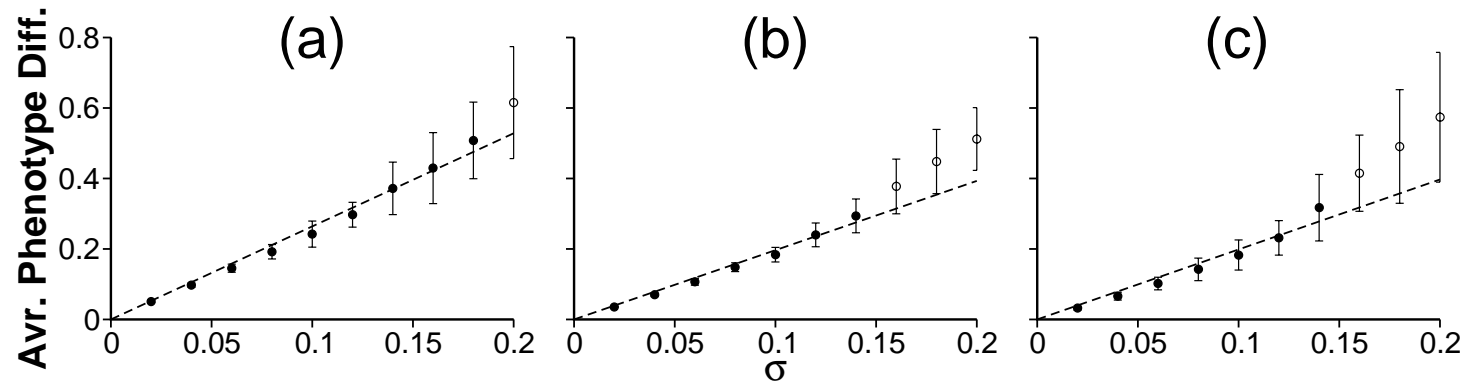